\title{
ESGE and ESGENA Position Statement on gastrointestinal endoscopy and the COVID-19 pandemic
}

Authors

Ian M. Gralnek ${ }^{*}$, , Cesare Hassan ${ }^{*}, 2$, Ulrike Beilenhoff ${ }^{3}$, Giulio Antonelli², Alanna Ebigbo4, Maria Pellisè, Marianna Arvanitakis $^{6}$, Pradeep Bhandari ${ }^{7}$, Raf Bisschops ${ }^{8}$, Jeanin E. Van Hooft ${ }^{9}$, Michal F. Kaminski ${ }^{10}$, Konstantinos Triantafyllou ${ }^{11}$, George Webster ${ }^{12}$, Heiko Pohl ${ }^{13}$, Irene Dunkley ${ }^{14}$, Björn Fehrke ${ }^{15}$, Mario Gazic ${ }^{16}$, Tatjana Gjergek ${ }^{17}$, Siiri Maasen ${ }^{18}$, Wendy Waagenes ${ }^{19}$, Marjon de Pater ${ }^{20}$, Thierry Ponchon ${ }^{21}$, Peter D. Siersema ${ }^{22}$, Helmut Messmann ${ }^{4}$, Mario Dinis-Ribeiro ${ }^{23}$

Institutions

1 Institute of Gastroenterology and Hepatology, Emek Medical Center, Afula, Israel \& Rappaport Faculty of Medicine Technion Israel Institute of Technology, Haifa, Israel

2 Nuovo Regina Margherita Hospital, Rome, Italy

3 Ulm, Germany

4 III. Medizinische Klinik Universitätsklinikum Augsburg, Augsburg, Germany

5 Department of Gastroenterology, Hospital Clinic de Barcelona. Institut d'Investigacions Biomediques August Pi i Sunyer (IDIBAPS). Centro de Investigación Biomédica en Red de Enfermedades Hepáticas y Digestivas (CIBERehd). Universitat de Barcelona, Barcelona, Spain

6 Department of Gastroenterology, Erasme University Hospital, Université Libre de Bruxelles, Brussels, Belgium

7 Portsmouth Hospital NHS Trust, Gastroenterology, Portsmouth, United Kingdom

8 Department of Gastroenterology and Hepatology, Catholic University of Leuven (KUL), TARGID, University, Hospitals Leuven, Leuven, Belgium

9 Department of Gastroenterology and Hepatology, Amsterdam University Medical Centers, University of Amsterdam, The Netherlands

10 Department of Cancer Prevention and Department of Oncological Gastroenterology, The Maria SklodowskaCurie National Research Institute of Oncology, Warsaw, Poland

11 Hepatogastroenterology Unit, 2nd Department of Internal Medicine - Propaedeutic, Medical School, National and Kapodistrian University of Athens, Attikon University General Hospital, Athens, Greece

12 Department of Gastroenterology, University College London Hospitals, London, United Kingdom
13 Dartmouth Geisel School of Medicine, Hanover NH, VA Medical Center, Section of Gastroenterology, White River Junction, VT., USA

14 North West Anglia NHS Foundation Trust, Hinchingbrooke, United Kingdom

15 Inselspital, Bern, Switzerland

16 General hospital, Bjelovar, Croatia

17 University Medical Centre, Ljubljana, Slovenia

18 Tallinn Healthcare College, Tallin, Estonia

19 Hvidovre Hospital, Copenhagen, Denmark

20 Amsterdam UMC location AMC, Amsterdam, The Netherlands

21 Gastroenterology Division, Edouard Herriot Hospital Lyon, France

22 Department of Gastroenterology and Hepatology, Radboud University Medical Center, Nijmegen, The Netherlands

23 Gastroenterology Department, Portuguese Oncology Institute of Porto, Portugal

Bibliography

DOI https://doi.org/10.1055/a-1155-6229

Published online: 17.4.2020 | Endoscopy 2020; 52: 483-490

(c) Georg Thieme Verlag KG Stuttgart · New York

ISSN 0013-726X

Corresponding author

Mario Dinis Ribeiro, Instituto Português de Oncologia -

Gastrenterologia, Rua Dr. Bernardino de Almedia,

Porto 4200-072, Portugal

mario@med.up.pt

\section{ABSTRACT}

We are currently living in the throes of the COVID-19 pandemic that imposes a significant stress on health care providers and facilities. Europe is severely affected with an exponential increase in incident infections and deaths. The clinical manifestations of COVID-19 can be subtle, encom-

* co-first authors 
passing a broad spectrum from asymptomatic mild disease to severe respiratory illness. Health care professionals in endoscopy units are at increased risk of infection from COVID-19. Infection prevention and control has been shown to be dramatically effective in assuring the safety of both health care professionals and patients. The European Society of Gastrointestinal Endoscopy (www.esge.com) and the European Society of Gastroenterology and Endoscopy Nurses and Associates (www.esgena.org) are joining forces to provide guidance during this pandemic to help assure the highest level of endoscopy care and protection against
COVID-19 for both patients and endoscopy unit personnel. This guidance is based upon the best available evidence regarding assessment of risk during the current status of the pandemic and a consensus on which procedures to perform and the priorities on resumption. We appreciate the gaps in knowledge and evidence, especially on the proper strategy (ies) for the resumption of normal endoscopy practice during the upcoming phases and end of the pandemic and therefore a list of potential research questions is presented. New evidence may result in an updated statement.

\section{Introduction}

The outbreak of COVID-19 disease has spread from its original cluster in Hubei province, China [1,2] throughout the world, and has been declared a pandemic by the World Health Organization [3]. Europe is severely affected with an exponential increase in the number of COVID-19 cases and deaths [4]. It has been estimated that approximately $10 \%$ of Health Care Professionals (HCP) have become COVID-19 positive in Western countries $[5,6]$. The clinical manifestations of COVID-19 are varied, encompassing a broad spectrum from asymptomatic mild disease, to severe critical respiratory illness leading to respiratory failure, multiorgan failure and death [1, 2, 7-9]. Thus, high clinical suspicion and appropriate risk stratification of patients are needed.

HCP in endoscopy units are at increased risk of infection by COVID-19 from inhalation of airborne droplets, conjunctival contact, and potential fecal-oral transmission [2,10]. Periendoscopic aerosolized infections have been reported, making upper Gl endoscopy a high-risk procedure [11 - 17]. In addition, live virus has been found in patient stool $[10,18-20]$. As a mechanism of entry, the angiotensin-converting enzyme II (ACE2) receptor, widely expressed in the intestinal tract [21], is likely used by the virus to enter human cells [2] making lower GI endoscopy procedures of uncertain risk status. Furthermore, infected HCP may transmit the infection to their colleagues, patients, families, and communities as hospital-based epidemics have been reported in European countries [22].

Infection prevention and control (IPC) has been shown to be dramatically effective in assuring the safety of both HCP and patients. This is not limited to the use of personal protective equipment (PPE), but is also based on a transparent and detailed IPC strategy, risk stratification of patients, correct use of PPE and interventions based on testing, separation and isolation of patients at high risk of COVID-19 [22-25].

Given the simultaneous COVID-19 outbreak in all European countries, a rational approach regarding limited resources is important $[22,26]$. Shortages do not only apply to PPE, but also to the availability of hospital infrastructure including HCP staff, availability of beds (including ICU beds), and medical equipment such as ventilators. On the other hand, the need to protect the patient population, especially patients at high risk of COVID-19 morbidity, has forced Endoscopy Units to post- pone a disproportionate number of procedures, weighing case-by-case the benefit of endoscopy with the risk of COVID19 infection. A clear and thoughtful policy regarding the timely rescheduling of these postponed endoscopy procedures will be required.

The European Society of Gastrointestinal Endoscopy (www. esge.com) and the European Society of Gastroenterology and Endoscopy Nurses and Associates (www.esgena.com) have joined forces to provide guidance in order to assure the highest level of protection against COVID-19 for both patients and health care personnel. This position statement was first presented as an online statement March 18, 2020 (www.esge.com and www.esgena.com) and is now updated.

This ESGE-ESGENA Position Statement will provide guidance on 4 main topics:

1. How to perform gastrointestinal ( $\mathrm{Gl}$ ) endoscopies during the COVID-19 viral pandemic?

2. Which GI endoscopy procedures should always be done? Which should be postponed?

3. How to protect $\mathrm{Gl}$ endoscopy unit personnel during the pandemic?

4. What knowledge is currently missing and what is needed in this evolving field?

\section{Methods}

A Pubmed/MEDLINE search was performed using 'severe acute respiratory distress syndrome coronavirus 2', 'COVID-19', 'endoscopy, digestive system endoscopy', 'gastrointestinal endoscopic examination, therapy' as MeSH terms. As our aim was to provide guidance rather than clinical recommendations, statements by international medical bodies such as the World Health Organization and the European and US Centers' for Disease Prevention and Control were prioritized. Furthermore, the most recent guidance available to date issued by major gastroenterology societies was reviewed.

Guidance was grouped according to the three main phases of an endoscopic procedure, that is, pre-, intra- and post-procedure.

In order to define the proper timing of endoscopy according to clinical indication, between the $23^{\text {rd }}$ and $25^{\text {th }}$ of March 2020 , the ESGE Governing Board addressed the main GI endoscopy 
Patient scheduled for Gl endoscopy procedure
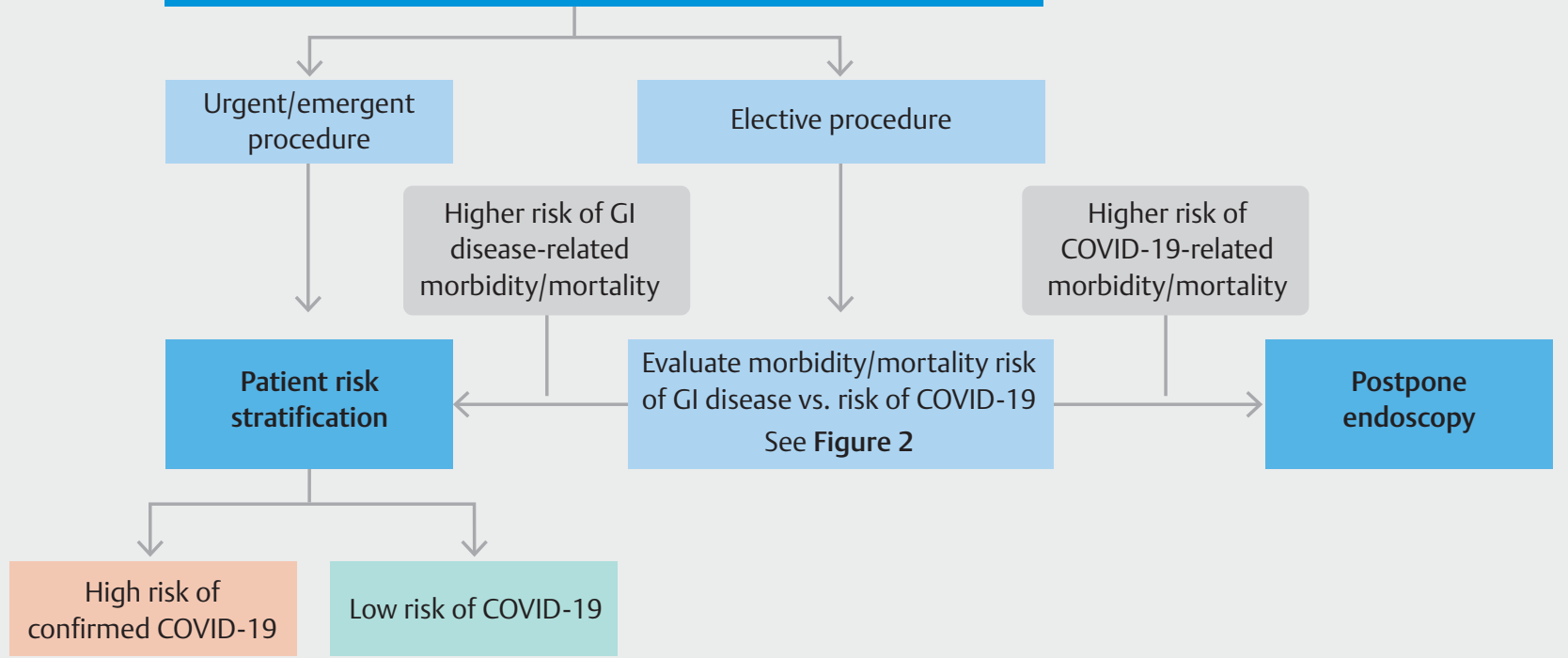

Appropriate PPE (see Table 2)

- Fig. 1 Decision pathway for GI endoscopic procedures during the COVID-19 pandemic. GI, gastrointestinal; PPE, personal protective equipment.

procedures, assigning them to predefined priority stratification groups and thereby differentiating between procedures that can be systematically performed or postponed, and those that must be assessed on a case-by-case basis weighing the tradeoffs of the medical indication / necessity with the COVID-19 risks. Endoscopic procedures were assigned to a group if an agreement/consensus of $\geq 75 \%$ was reached. When agreement/consensus was not reached, the endoscopic procedure was recommended to be performed on a case-by-case basis.

All Governing Board members were also asked to vote on the level of priority for rescheduling postponed endoscopic procedures (high or low priority) and to assign every endoscopic procedure a proposed rescheduling time (in weeks).

\section{Part I:}

\section{How to perform gastrointestinal (GI) endoscopies during the COVID-19 viral \\ pandemic}

\section{General comments}

1. The entire staff of the endoscopy unit must be appropriately trained and informed on the IPC strategy for COVID19 [22]. This should include potential sources of contamination, hygiene measures, COVID-19 risk factors, correct use of PPE, and interventions, such as separation, isolation and testing, for high-risk or infected patients.

2. Health Care Professionals (HCP) in endoscopy units should be triaged daily: staff should assess themselves according to potential risk factors, symptoms and signs (daily measurement of temperature). Those considered to be at high-risk of COVID-19 should be isolated and tested.

3. COVID-19 can effectively be inactivated by commonly used disinfectants having virucidal activity (EN 14885). Reprocessing of flexible endoscopes and endoscopic accessories should be performed according to published guidelines [28]. Reuse of any disposable GI endoscopic device is strongly discouraged. During reprocessing, mucosal surfaces must be protected as recommended [28]. Additional precautions should be taken in the reprocessing of equipment, such as FFP2/3 masks, after endoscopy in confirmed COVID-19 cases.

4. Each GI endoscopy unit should have a detailed plan for the cleaning and disinfecting of endoscopy procedure rooms

[29]. Cleaning the endoscopy unit with virucidal agents is recommended as infection by contact is possible. This is mandatory after each case in patients at high-risk of or known infection with COVID-19 [29].

5. If feasible, online care should be provided (e.g. telemedicine). If this is to replace an outpatient clinic visit, audio and video transmission is preferred and formal documentation in the patient's medical record should be performed.

6. Washing of hands with soap and warm water (for at least 20seconds) or use of alcohol-based hand rub, before and after all patient interactions, after contact with potentially infectious sources, and before and after gowning, should be done by all $\mathrm{Gl}$ endoscopy unit personnel. Mobile phones, pens, computer workstations, and medical equipment should not be shared. Jewelry (watches, rings, bracelets) should not be worn by GI endoscopy unit HCP. 


\section{Pre-procedure Risk Management}

1. Risk stratification of patients for possible COVID-19 infection should be done 1 day prior to GI endoscopy (by phone preferably) and then again on the day of endoscopy [13, 30, 31 ] by questioning for symptoms and contacts; or if / when available through tests for virus infection or immunity

(- Fig. 1).

- Please note that:

- Given the current pandemic status of COVID-19 (April 2020), it may be reasonable according to the local situation, that all patients be considered at high-risk for COVID-19 infection and proper measures should be taken to protect HCP.

- In the different phases of this pandemia, based on epidemiological factors, testing and potential immunity, patient risk stratification may be possible, converting previously high-risk patients to low-risk.

2. During patient assessment on the day of endoscopy, use of surgical masks is recommended for both the HCP and the patient and a distance of at least $1-2$ meters is recommended, as well as the use of a physical barrier, such as glass or face-shield, if possible. Before entering the $\mathrm{GI}$ endoscopy unit, temperature measurement should be performed on all patients.

3. Relatives and caregivers should not have access to the GI endoscopy unit. If it is exceptionally required, they should undergo the same risk assessment as the patient.

4. For patients who are considered at high risk for COVID19 , separate pre- and post-GI endoscopy recovery areas (or timeslots) should be arranged.

5. Whenever possible, all patients entering the $\mathrm{Gl}$ endoscopy unit should wear respiratory protective equipment (facial mask).

\section{Intra-procedure risk management}

1. During the current situation in most countries, only essential and fully trained endoscopy personnel should be present in endoscopy cases, all using a full set of PPE. Training programs should be adjusted during this phase, and the use of e-learning is to be encouraged.

2. According to the patient's risk status, PPE should include gloves, hairnet, protective eyewear (goggles or face shield), waterproof gowns, booties/shoe covers, and respiratory protective equipment. High-filter respiratory masks (FFP2/ 3 ) and booties/shoe covers should be used for high-risk or infected cases $[13,22,25]$ ( $\triangleright$ Table 2 ). Putting on and taking off PPE must be performed as recommended [32] - see also ESGENA-Poster www.esgena.org. In situations of limited availability of masks/respiratory protective equipment, a protective face shield is a useful alternative tool. Prolonged use of face masks/respiratory protective equipment of up to 4 hours is acceptable.

3. Although different GI endoscopic procedures may have different levels of risk, for the sake of simplicity and safety we recommend the same personal protection measures for all procedures, both upper or lower GI endoscopies [25, 33, 34].
- Table 1 Risk stratification for potential COVID-19 infection in patients requiring gastrointestinal endoscopy.

Low-risk No symptoms (eg, cough, fever, shortness of breath or patient diarrhea)

AND

No history of contact with COVID positive individual AND

No travel or residence in a location reporting community transmission of COVID-19 during previous 14 days Negative testing for COVID 19 (with adequate accuracy and proper epidemiological setting)

High-risk Presence of symptoms with adequate sensitivity (eg, patient cough, fever, shortness of breath or diarrhea) OR

Travel or residence in a location reporting community transmission of COVID-19 during previous 14 days (eg, most European regions in April 2020) OR

Contact with COVID-19 positive (or very likely to be positive) individual

- Table 2 Health-professional personal protective equipment stratified by patient risk

\begin{tabular}{|l|l|}
\hline Low-Risk Patient & High-risk or Positive patient \\
\hline Surgical mask & $\begin{array}{l}\text { Respiratory PPE (FFP2/ } \\
\text { FFP3 mask) }\end{array}$ \\
\hline Gloves $^{3}$ & Two pairs of gloves \\
\hline Booties/shoe covers & Booties/shoe covers \\
\hline Disposable hairnet & Disposable hairnet \\
\hline $\begin{array}{l}\text { Protective eyewear (goggles } \\
\text { or disposable face shield) }\end{array}$ & $\begin{array}{l}\text { Protective eyewear (goggles or } \\
\text { disposable face shield) }\end{array}$ \\
\hline $\begin{array}{l}\text { Water-proof disposable gowns } \\
{ }^{4}\end{array}$ & Water-proof disposable gowns \\
\hline${ }^{1}$ DIN EN 14683:2019-6 \\
${ }^{2}$ DIN EN 149:2001-10 \\
${ }^{3}$ DIN EN 420/DIN EN 374 \\
${ }^{4}$ DIN EN 14126:2004-01
\end{tabular}

4. Whenever possible, in patients who are considered to be at high risk or who are known to be positive for the COVID-19 virus, Gl endoscopy should be performed only if medically indicated and if available, in a negative-pressure room by experienced staff [29]. If the only negative-pressure rooms are located outside the endoscopy unit, it must be ensured that these rooms are properly equipped for performing any Gl endoscopy procedure. If negative-pressure rooms are not available, endoscopy should be performed in a dedicated room with adequate ventilation. All the other a forementioned protective measures should be taken and the risk of postponing endoscopy versus the risk of COVID-19 infection should be considered.

5. For patients in intensive care units (ICUs), GI endoscopy should be performed bedside. 


\section{Post-procedure risk management}

1. Consider tracing and contacting patients at 7 and 14 days to inquire about any new COVID-19 diagnosis, or development of COVID-19 symptoms.

2. Contaminated waste and endoscopic devices from patients at high risk of or with suspected or confirmed COVID-19 should be disposed of using the specific local regulations related to high-risk waste management.

Part II:

Timing of endoscopy during the COVID-19 pandemic according to medical indication

1. Gl endoscopy units should strongly consider temporarily postponing elective, non-urgent endoscopy procedures, based upon availability of local human resources and local policies that may depend on regional/national pandemic rules/regulations ( $>$ Fig. 1 and $\triangleright$ Fig. 2 )

2. The following list of $\mathrm{Gl}$ endoscopy procedures should always be performed ( $\triangleright$ Fig. 2 )

- Acute upper/lower GI bleeding with hemodynamic instability

- Capsule/enteroscopy for urgent/emergent bleeding

- Anemia with hemodynamic instability

- Foreign body in esophagus and/or high-risk foreign body in the stomach

- Obstructive jaundice

- Acute ascending cholangitis

3. During the current COVID-19 pandemic, the following list of Gl endoscopy procedures should be postponed with no need to reschedule before 12 weeks (low priority)

( Fig. 2)

- Surveillance for:

- Barrett's Esophagus without dysplasia or Low-Grade Dysplasia or after endoscopic treatment

- Gastric atrophy/Intestinal Metaplasia

- Inflammatory Bowel Disease

- Primary Sclerosing Cholangitis

- Post-endoscopic resection (including immediate endoscopy after resection), surgical resection of cancer or post-polypectomy surveillance

- Diagnosis/surveillance of Lynch syndrome and other hereditary syndromes

- Diagnosis of Irritable Bowel Syndrome-like symptoms

- Diagnosis of reflux disease, dyspepsia (no alarm symptoms)

- Screening in high risk patients for esophageal cancer, gastric cancer, colon cancer (primary screening endoscopy) or pancreatic cancer

- Bariatric Gl endoscopy procedures (e.g., intra-gastric balloons, endoscopic sleeve gastroplasty)
4. Each of the following Gl endoscopy procedures warrant a case-by-case evaluation based upon medical necessity ( Fig.2). In general, therapeutic endoscopic procedures or those affecting prognosis (and whenever further therapies can be assured), namely those that are cancer-related or severely symptomatic, should be ranked as "high-priority" (either to be performed immediately or postponed within 12 weeks). All others "low priority" may be either performed immediately or post-poned to beyond 12 weeks on a case-by-case assessment.

\section{High-priority endoscopy procedures}

- Endoscopic treatment of high-grade dysplasia (HGD) or early intra-mucosal cancer in the esophagus, stomach, or large colonic polyps at high-risk of submucosal invasion

- Malignant stricture stenting

- PEG/PEJ/NJ tube

- Upper GI fistula/leakage

- Dysphagia or dyspepsia with alarm symptoms present

- Upper GI bleeding without hemodynamic instability

- Rectal bleeding

- Colonoscopy for melena after negative upper-GI endoscopy

- Severe anemia with no hemodynamic instability

- Tissue acquisition needed for the initiation of systemic therapy/surgery

- Colonoscopy within organized FOBT + CRC screening programme

- Foreign body in the stomach, low-risk

- Benign stricture requiring dilation/stenting

- Radiologic evidence of mass

- Lymph node EUS sampling

- Gallstone-related pancreatitis

- Pancreatic mass/stricture

- Biliary stricture dilation

- Pancreatico-biliary stent replacement for non-urgent indication

- Necrosectomy

\section{Low-priority endoscopy procedures}

- Endoscopic treatment of esophageal or gastric low-grade dysplasia (LGD)

- Duodenal polyp

- Ampullectomy

- Band ligation/non-emergency

- Iron deficiency anemia

- Pancreatic cyst (depending on risk features)

- Biliary stricture/no urgency (no cholangitis, no jaundice, etc.)

- Submucosal lesion EUS sampling

- Achalasia (POEM, balloon dilitation)

- gFOBT/FIT + (outside of an organized regional/national screening program)

\section{Conclusion}

The COVID-19 pandemic is having a disruptive effect on the workflow and safety of Gl-endoscopy units worldwide. Most GI endoscopy units in Europe are having to manage the current si- 
Perform always

- Acute upper/lower GI bleeding with hemodynamic instability

- Capsule/enteroscopy for urgent/emergent bleeding

- Anemia with hemodynamic instability

- Foreign body in esophagus and/or high-risk foreign body in the stomach

- Obstructive jaundice

- Acute ascending cholangitis

\section{Case by case management - high priority}

- Endoscopic treatment of high-grade dysplasia (HGD) or early intramucosal cancer in the esophagus, stomach, or large colonic polyps at high-risk of submucosal invasion

- Malignant stricture stenting

- PEG/PEJ/NJ tube

- Upper Gl fistula/leakage

- Dysphagia or dyspepsia with alarm symptoms present

- Upper GI bleeding without hemodynamic instability

- Rectal bleeding

- Colonoscopy for melena after negative upper-Gl endoscopy

- Severe anemia with no hemodynamic instability

- Tissue acquisition needed for the initiation of systemic therapy/surgery

- Colonoscopy within organized FOBT+ CRC screening programme

- Foreign body in the stomach, low-risk

- Benign stricture requiring dilation/stenting

- Radiologic evidence of mass

- Lymph node EUS sampling

- Gallstone-related pancreatitis

- Pancreatic mass/stricture

- Biliary stricture dilation

- Pancreatico-biliary stent replacement for non-urgent indication

- Necrosectomy

\section{Case by case management - low priority}

- Endoscopic treatment of esophageal or gastric low-grade dysplasia (LGD)

- Duodenal polyp

- Ampullectomy

- Band ligation/non-emergency

- Iron deficiency anemia

- Pancreatic cyst (depending on risk features)

- Biliary stricture/no urgency (no cholangitis, no jaundice, etc.)

- Submucosal lesion EUS sampling

- Achalasia (POEM, balloon dilitation)

- gFOBT/FIT+ (outside of an organized regional/ national screening program)

Postpone always

- Surveillance for

- Barrett's Esophagus without dysplasia or Low-Grade Dysplasia or after endoscopic treatment

- Gastric atrophy/Intestinal Metaplasia

- Inflammatory Bowel Disease

- Primary Sclerosing Cholangitis

- Post-endoscopic resection (including immediate endoscopy after resection), surgical resection of cancer or post-polypectomy surveillance

- Diagnosis/surveillance of Lynch syndrome and other hereditary syndromes

- Diagnosis of Irritable Bowel Syndrome-like symptoms

- Diagnosis of reflux disease, dyspepsia (no alarm symptoms)

- Screening in high risk patients for esophageal cancer, gastric cancer, colon cancer (primary screening endoscopy) or pancreatic cancer

- Bariatric Gl endoscopy procedures (e.g., intra-gastric balloons, endoscopic sleeve gastroplasty)

- Fig. 2 List of indications for endoscopic procedures according to rescheduling recommendations and priority.

tuation with shortages of personnel and PPEs, substantial reductions in the volume of screening endoscopy procedures, enormous pressures on prioritizing endoscopic procedures, and postponing many procedures without knowing exactly when patients will be rescheduled.

Gl endoscopy units should consider this ESGE position statement against local rules and recommendations. Also, due to the COVID-19 pandemic, individual clinical judgment and local re- sources may lead to alternative perspectives in regard to which procedures/patients are to be prioritized and when to resume a more regular endoscopy procedure schedule. It should be noted that $54 \%$ of survey respondents reported that the decision to postpone $\mathrm{Gl}$ endoscopy procedures was related to lack of PPE. This must command our community's full attention for future outbreaks or similar emergent medical situations. 
- Table 3 Suggested Research Agenda

Infection and workflow/unit/staff

- How to consider the lingering effects of COVID 19 during the coming months/years in our endoscopy practice?

- When and how should a patient suspected of having COVID 19 be tested in relation to performance of a $\mathrm{Gl}$ endoscopy procedure?

- How often, or if at all,should medical staff/endoscopy staff be tested for COVID 19 and by which methods?

- How did COVID-19 affect the endoscopy unit's workflow?

- How to take care of the psychological well-being of the GI endoscopy unit staff?

- What are the financial consequences of the COVID-19 outbreak for the endoscopy unit?

- How did COVID-19 affect fellows' training, education, and research (meeting, e-learning, CME credits, collaborations, etc.)?

- How to stimulate/compensate the staff to work extra hours to catch up with the patient waiting lists after the pandemic?

\section{Procedural protection}

- Is there any difference in COVID 19 transmission risk between upper and lower $\mathrm{Gl}$ endoscopy?

- Is oral and/or fecal transmission a true/equal hazard?

- Which are the fundamental PPEs that are required and how to confront their shortages?

- What is the difference in using a FFP2 vs two surgical masks vs one surgical mask on infection risk?

\section{Rescheduling and disease risk}

- What is the burden in terms of cancer progression of delaying $\mathrm{Gl}$ endoscopy procedures due to the COVID-19 pandemic?

- How did you organize the Gl endoscopy care for patients?

- How to prioritize postponed Gl endoscopy procedures after the pandemic is over?

- What are the "acceptable" waiting times, stratified by the type of Gl endoscopy procedure?

We also believe that further research is urgently needed in order to clarify the overall burden of COVID-19 on our GI endoscopy units but also in relation to how to effectively run endoscopic units during and after this pandemic ( $\triangleright$ Table 3 ).

\section{Competing interests}

I. M. Gralnek has received lecture fees from Astra-Zeneca, Taro Pharma, Vifor Pharma and 3D Matrix (ongoing); consultant fees from Boston Scientific, Gl view, Motus Gl and Symbionix (ongoing); and is DSMB Member by Intec Pharma, MAB member by Motus GI. J. E. van Hooft has received lecture fees from Medtronics (2014 - 2015) and Cook Medical (2019), and consultancy fees from Boston Scientific (2014 - 2017); her department has received research grants from Cook Medical (20142019) and Abbott (2014-2017). M. Kaminski has received lecture fees from Boston Scientific (2018 - ongoing) and consultancy fees from Olympus and Fujifilm (2018-ongoing). P. D. Siersema has received research grants from Norgine, Pentax, Microtech, Yakult and Motus $\mathrm{Gl}$ (ongoing) and is on the Advisory Board of Motus Gl. G. Antonelli, C.
Hassan, A. Ebigbo, M. Pellisé, M. Arvanitakis, P. Bhandari, R. Bisschops, K. Triantafyllou, G. Webster, H. Pohl, T. Ponchon, H. Messmann, M. Dinis-Ribeiro have no competing interest.

\section{References}

[1] Guan W-J, Ni Z-Y, Hu Y et al. Clinical Characteristics of Coronavirus Disease 2019 in China. N Engl J Med 2020: doi:10.1056/NEJMoa2002032

[2] Huang C, Wang Y, Li X et al. Clinical features of patients infected with 2019 novel coronavirus in Wuhan, China. Lancet 2020; 395: 497-506

[3] WHO Director-General's opening remarks at the media briefing on COVID-19. https://www.who.int/dg/speeches/detail/who-directorgeneral-s-opening-remarks-at-the-media-briefing-on-covid-19-11march-2020

[4] Download today's data on the geographic distribution of COVID-19 cases worldwide. European Centre for Disease Prevention and Control; 2020: https://www.ecdc.europa.eu/en/publications-data/download-todays-data-geographic-distribution-covid-19-cases-worldwide

[5] Remuzzi A, Remuzzi G. COVID-19 and Italy: what next?The Lancet; 2020: https://www.thelancet.com/journals/lancet/article/PIIS01406736(20)30627-9/abstract

[6] Ng K, Poon BH, Kiat Puar TH et al. COVID-19 and the Risk to Health Care Workers: A Case Report. Ann Intern Med; 2020: https://annals. org/aim/fullarticle/2763329/covid-19-risk-health-care-workers-casereport

[7] Wang D, Hu B, Hu C et al. Clinical Characteristics of 138 Hospitalized Patients With 2019 Novel Coronavirus-Infected Pneumonia in Wuhan, China. [Epub ahead of print]. JAMA 2020: doi:10.1001/ jama.2020.1585

[8] Wu C, Chen X, Cai Y et al. Risk Factors Associated With Acute Respiratory Distress Syndrome and Death in Patients With Coronavirus Disease 2019 Pneumonia in Wuhan, China. JAMA Intern Med. 2020

[9] Young BE, Ong SWX, Kalimuddin S et al. Epidemiologic Features and Clinical Course of Patients Infected With SARS-CoV-2 in Singapore. JAMA. 2020

[10] Gu J, Han B, Wang J. COVID-19: Gastrointestinal manifestations and potential fecal-oral transmission. [Epub ahead of print]. Gastroenterology 2020: doi:10.1053/j.gastro.2020.02.054

[11] Tang Jw, Li Y, Eames I et al. Factors involved in the aerosol transmission of infection and control of ventilation in healthcare premises. J Hosp Infect 2006; 64: 100-114

[12] Parodi SM, Liu VX. From Containment to Mitigation of COVID-19 in the US. JAMA; 2020: https://jamanetwork.com/journals/jama/fullarticle/2763187

[13] Repici A, Maselli R, Colombo M. Coronavirus (COVID-19) outbreak: what the department of endoscopy should know. Gastrointestinal Endoscopy; 2020: https://www.giejournal.org/article/S0016-5107 (20)30245-5/abstract

[14] Lui RN, Wong SH, Sánchez-Luna SA et al. Overview of guidance for endoscopy during the coronavirus disease 2019 (COVID-19) pandemic. [Epub ahead of print]. J Gastroenterol Hepatol 2020: doi: $10.1111 /$ jgh. 15053

[15] Sultan S, Lim JK, Altayar O. AGA Institute Rapid Recommendations for Gastrointestinal Procedures During the COVID-19 Pandemic. Gastroenterology; 2020: https://www.gastrojournal.org/article/S00165085(20)30458-3/abstract

[16] JOINT GASTROENTEROLOGY SOCIETY MESSAGE: COVID-19 Use of Personal Protective Equipment in GI Endoscopy. https://www.asge. org/home/advanced-education-training/covid-19-asge-updates-formembers/joint-gastroenterology-society-message-covid-19-use-ofpersonal-protective-equipment-in-gi-endoscopy 
[17] Endoscopy activity and COVID-19: BSG and JAG guidance - update 03.04.20. The British Society of Gastroenterology; 2020: https:// www.bsg.org.uk/covid-19-advice/endoscopy-activity-and-covid-19bsg-and-jag-guidance/

[18] Xiao F, Tang M, Zheng $X$ et al. Evidence for gastrointestinal infection of SARS-CoV-2. [Epub ahead of print]. Gastroenterology 2020: doi:10.1053/j.gastro.2020.02.055

[19] Kanne JP. Chest CT Findings in 2019 Novel Coronavirus (2019-nCoV) Infections from Wuhan, China: Key Points for the Radiologist. Radiology 2020; 295: 16-17 doi:10.1148/radiol.2020200241

[20] Song Y, Liu P, Shi XL. SARS-CoV-2 induced diarrhoea as onset symptom in patient with COVID-19. Gut; 2020: https://gut.bmj.com/content/early/2020/03/16/gutjnl-2020-320891

[21] Hamming I, Timens W, Bulthuis MLC. Tissue distribution of ACE2 protein, the functional receptor for SARS coronavirus. A first step in understanding SARS pathogenesis. J Pathol 2004; 203: 631-637

[22] Infection prevention and control and preparedness for COVID-19 in healthcare settings. European Centre for Disease Prevention and Control; 2020: https://www.ecdc.europa.eu/en/publications-data/ infection-prevention-and-control-and-preparedness-covid-19healthcare-settings

[23] Guidance for wearing and removing personal protective equipment in healthcare settings for the care of patients with suspected or confirmed COVID-19. https://www.ecdc.europa.eu/en/publicationsdata/guidance-wearing-and-removing-personal-protective-equipment-healthcare-settings

[24] Zhang $Y$, Zhang X, Liu L et al. Suggestions for infection prevention and control in digestive endoscopy during current 2019-nCoV pneumonia outbreak in Wuhan, Hubei province, China. Endoscopy 2020; 52: 312-314

[25] Infection prevention and control during health care when novel coronavirus (nCoV) infection is suspected. https://www.who.int/publications-detail/infection-prevention-and-control-during-health-carewhen-novel-coronavirus-(ncov)-infection-is-suspected-20200125
[26] Cloth masks and mask sterilisation as options in case of shortage of surgical masks and respirators. European Centre for Disease Prevention and Control; 2020: https://www.ecdc.europa.eu/en/publications-data/cloth-masks-sterilisation-options-shortage-surgicalmasks-respirators

[27] GI Endoscopy Activity and COVID-19: Next steps - Updated 03.04.20. The British Society of Gastroenterology; 2020: https://www.bsg.org. uk/covid-19-advice/gi-endoscopy-activity-and-covid-19-next-steps/

[28] Beilenhoff U, Biering H, Blum R et al. Reprocessing of flexible endoscopes and endoscopic accessories used in gastrointestinal endoscopy: Position Statement of the European Society of Gastrointestinal Endoscopy (ESGE) and European Society of Gastroenterology Nurses and Associates (ESGENA) - Update 2018. Endoscopy 2018; 50: 12051234

[29] Calderwood AH, Day LW, Muthusamy VR. ASGE Quality Assurance in Endoscopy Committee. et al. ASGE guideline for infection control during Gl endoscopy. Gastrointest Endosc 2018; 87: 1167-1179

[30] Recomendaciones de la SEED: Protección en Unidades de Endoscopia frente al COVID-19. https://wseed.es/images/site/guia_clinica/2020/ RecomendacionesSEED_ProteccionUnidadesEndoscopia_Coronavirus.pdf

[31] Epidémie de COVID-19: recommandations en endoscopie digestive. SFED; 2020: https://www.sfed.org/professionnels/actualites-pro/epidemie-de-covid-19-recommandations-en-endoscopie-digestive

[32] CDC sequence for putting on personal protective equiment (PPE). https://www.cdc.gov/hai/pdfs/ppe/ppe-sequence.pdf

[33] van Doremalen N, Bushmaker T, Morris DH et al. Aerosol and Surface Stability of SARS-CoV-2 as Compared with SARS-CoV-1. [Epub ahead of print]. New England Journal of Medicine 2020: doi:10.1056/ NEJMc2004973

[34] Brewster D], Chrimes NC, Do TB et al. Consensus statement: Safe Airway Society principles of airway management and tracheal intubation specific to the COVID-19 adult patient group. Med ] Aust; Published online: 16 March 2020. 